\title{
WSAA 8
}

P. Houston; L. O'Raifeartaigh

On the charge distribution of static axial and mirror symmetric monopole systems

In: Zdeněk Frolík (ed.): Abstracta. 8th Winter School on Abstract Analysis.

Czechoslovak Academy of Sciences, Praha, 1980. pp. 96--103.

Persistent URL: http://dml.cz/dmlcz/701186

\section{Terms of use:}

(C) Institute of Mathematics of the Academy of Sciences of the Czech Republic, 1980

Institute of Mathematics of the Academy of Sciences of the Czech Republic provides access to digitized documents strictly for personal use. Each copy of any part of this document must contain these Terms of use.

This paper has been digitized, optimized for electronic delivery
and stamped with digital signature within the project $D M L-C Z$ :
The Czech Digital Mathematics Library http://project.dml.cz


EIGHTH WINTER SCHOOL ON ABSTRACT ANALYSIS (1980)

On the Charge Distribution of Static Axial and Mirror Symmetric Monopole Systems

P. Houst on and L. O'Ra1feartalgh

It has been shown that the Imposition of axial and mirror symmetry on the static magnetic SU(2) Yang-Mille-Higge oystem reduces the usual first order ("eelf-dual") Bogomolny equat1ons to five equations for five unknown (gauge-invariant) functions. In this note we show that, for a differentiable Higgs field, the five field equations and the topology rule out all charge-dietributions along the axis of symmetry except a single-point distribution, and the boundary conditions at the single point distribution are discussed.

Ever since the discovery [1] that the static Yang-Mills-Higgs equations of unified gauge-theory adnit finite-energy magnetic monopole solutions, there has been interest in the question of solutions which go beyond the original ones, which are all spherically symmetric. The next simplest configuration seems to be that in. which the fields are axially symmetric and symmetric under reflexions in all planes containing the zaxis, and In this note we wish to consider the field equations for such systems.

To obtain the simplest possible system of this kind we do not consider the full second-order MiH equations but rather the first-order equations

$$
\underset{\sim}{F}=\underset{\sim}{D} \text {. }
$$


which are obtained by minimizing the Bogomolny-Prasad-Sommerfield [2] Hamiltonian

$$
H=\frac{1}{2} \int d_{3} \times\left(F_{M}^{2}+\left(D_{M} \Phi\right)^{2}\right) .
$$

In which the gauge-group is SU(2), the Higgs field $\Phi$ 18 assumed to belong to the adjoint representation, and the ecalar potential 18 set equal to zero and replaced by the boundary condition $(\Phi, \Phi) \rightarrow c^{2}$ as $r \rightarrow \infty$ :

It has been shown $[3,4]$ that for axial and mirror symmetry. the field equations (1) reduce to the five equations

$$
\begin{aligned}
& k u_{\alpha}=b_{1 \alpha}+\rho \in_{\alpha \beta} \dot{h}_{1 \beta} . \\
& -k_{1 \alpha}=b u_{\alpha}+\rho h \in_{\alpha \beta} \dot{u}_{\beta} \\
& h k=\rho \in_{\alpha \beta} u_{\alpha \beta} .
\end{aligned}
$$

for the five (gauge-invariant) unknown functions $h, k, b, u_{\alpha}$ defined as

$$
\begin{gathered}
h^{2}=(\Phi, \Phi) \cdot h^{2} k^{2}=\left(D_{\psi} \Phi, D_{\psi} \Phi\right), \\
h^{2} k^{2}\left(b^{2}+k^{2}\right)=\left(D_{\psi}^{2} \Phi, D_{\psi}^{2} \Phi\right): h_{1 \alpha} h_{,}+h^{2} u_{\alpha} u_{\beta}= \\
=\left(D_{\alpha} \Phi, D_{\beta} \Phi\right)^{\cdot} .
\end{gathered}
$$

It was also shown that the first. four equations are equivalent.

to the existence of a master-potential $W$ such that

$$
\begin{aligned}
& \frac{\partial w}{\partial z}=h b, h^{2}=c^{2}+\Delta w \\
& 2 \rho \frac{\partial w}{\partial \rho}=\rho^{2}\left(h^{2}-c^{2}\right)+n^{2}-k^{2}-b^{2} .
\end{aligned}
$$

In particular

$$
\left(k^{2}+b^{2}\right)_{, \alpha}-\rho^{2}\left(h^{2}\right)_{, \alpha}-2 \rho \in_{\alpha \beta}(b h), \beta
$$

Furthermore, the ordinary Maxwell magnetic field $\mathrm{B}$. defined as usual $[5]$. 'to be

$$
B_{1}=\left(F_{1}, \phi\right)-\frac{1}{2} \epsilon_{1 j k}\left(\phi, D_{j} \phi \wedge D_{k} \phi\right) \text {, where } \phi=\Phi / h \text {. }
$$

turns out to be just.. 
(10)

$$
B_{\alpha}=\frac{1}{\rho} \epsilon_{\alpha \beta} b_{1 \beta} \quad,\left(B_{\gamma}=0\right) \text {. }
$$

Thus the magnetic charge contained in any volume of revolution $\checkmark$ around the z-axis and intersecting the axis at two points $z_{1}$ and $z_{2} 18$ just

$$
\Delta n=\frac{1}{4 \pi} \int d_{3} \times \nabla_{m} \min _{m}=\frac{1}{2}\left(b\left(z_{1}\right)-b\left(z_{2}\right)\right) \text {. }
$$

Of course, since (8) is a curl the charge can be located only at points where 18 not well defined 1.0. where the Higge fleld $\Phi$ is zero. The boundary conditions for the functions $h, b, k$. $u_{\alpha}$ as $r \rightarrow 0$ arie

$$
k, u_{\alpha} \rightarrow 0, h \rightarrow c-\frac{n}{r}, b \rightarrow-n \cos \theta .
$$

where $n$ 1o the total topological charge (In agreement with (9)). The boundary conditions on the zeaxis are determined by the finiteness of the energy [2]. which in terms of the var1ables $h, k, b, u_{\alpha}$ takes the form

$$
\begin{aligned}
& H=\frac{1}{2} \int d_{3} x\left[\left(\nabla_{m}\right)^{2}+h^{2} u_{w}^{2}+\left(\in_{\alpha \beta} u_{\alpha, \beta}\right)^{2}+\right. \\
& \left.+\frac{1}{\rho^{2}}\left\{h^{2} k^{2}+\left(b_{, \alpha}-k u_{\alpha}\right)^{2}+\left(k, \alpha+b u_{\alpha}\right)^{2}\right\}\right] .
\end{aligned}
$$

From this equation we see that (subject to uniform behaviour with respect to $z$ ) we have

$$
\rho_{\text {as }}^{\rho^{3 / 2} \nabla_{h} \rightarrow 0} \rightarrow 0: u_{\alpha} \rightarrow b^{-1} k_{1 \alpha} \rightarrow-k^{-1} b_{1 \alpha} . h k \rightarrow 0 .
$$

From the central equation in (14). and from (10) we have in particular

$$
b^{2}+k^{2}=n^{2} \text { for } \rho=0 \text {. }
$$

This equation also follows from $(3,4,5)$ and the first eqn. In (14).

Iń a previous note [6] it was shown that topological conoiderations alone implied that for an axially eymmetric oystem 
with charges located on the z-axis, the Higgs field $h$ had to vanish everywhere along the axis joining these points, and it is interesting to note that this result can be verified directly from (14) and (15) in a gauge-invariant manner. For if $h \neq 0$ on the axis, then from (14) we have $k=0$ and hence from (15) $b= \pm n$. It then follows from (11) that between any two points where $h \neq 0$ the charge must be either 0 , or $n$. Since $n$ is the total charge this establishes the result. The fact that the Higgs field must vanish betweencharges suggests that actually there can be no such distribution, except at a single point, and we now wish to show that, provided that the Higgs field $h$ has a continuous derivative such is indeed the case. Although this condition is quite mild, it is a little more than is required by finite energy.

The basis for our result will be the two equations

$$
\begin{aligned}
& \Delta(h b)=\left(h^{2}\right), z . \\
& \Delta(h k)=\frac{2\left(f f, \alpha-g \in_{\alpha \beta} f, \beta\right)}{h^{2} D^{2}}(h k), \alpha+ \\
&+\left[\frac{D^{2}}{\rho^{2}}+\frac{2 \epsilon_{\alpha \beta} g, \alpha f, \beta}{h^{2} D^{2}}\right](h k) .
\end{aligned}
$$

$$
f \equiv \rho h^{2}, g \equiv h b \cdot D^{2}=b^{2}+k^{2}+\rho^{2} h^{2} \text {. }
$$

which follow directly from (3) - (7). If we now assume that at any zero, $z_{0}$. of the Higgs field on the z-axis, $h$ is $c^{1}$ then (17) has the small distance approximation

$$
\Delta(h k)=\frac{n^{2}}{\rho^{2}}(h k) \text {. }
$$

Since finite energy requires that $h k \rightarrow 0$, as $\underset{m}{x \rightarrow z}$, the solution of (19) is an associated Legendre function of the first kind. Thus wherever $P_{m}^{n}(\cos \theta) \neq 0$ we have 
(20)

$$
h k \rightarrow h r m_{m}^{n}(\cos \theta), \quad m \geq n,
$$

where $r, \theta$ are polar coordinates about $z_{m}$. Since $k$ is bounded, equation (20) already shows that $h$ does not vanish infinitely fast at $z_{m}$, but between the powers $r$ and $r^{m}$.

Let us now consider those points $z_{\text {mo }}$, where $h \rightarrow 0$, but $b \neq 0$ for small $r$ and either $\theta=0$ or $\pi$ (or both). It follows from (14) and (15) that such points exist. Also from (15) we see that, as $r \rightarrow 0$,

$$
(h b)^{2}+(h k)^{2} \rightarrow n^{2} k \text {. }
$$

and that, since $b \nrightarrow 0$, hk can not dominate hb, for all $\theta$. From (21) we can write (16) in the form

$$
\Delta(h b)=\frac{1}{n^{2}}\left[\left(h^{2} b^{2}\right)_{1 z}+\left(h^{2} k^{2}\right), z\right]+\cdots \cdot
$$

Since $h k$ does not dominate $h b$ and $h b \rightarrow 0$ as $r \rightarrow 0$, it follows from (22) that the leading behaviour of hk is a solution of the homogeneous equation $\Delta(h b)=0$. In other words

$$
h b \rightarrow K_{s} r^{s} P_{s}(\cos \theta) \quad \text { for some } s \leq m \text {. }
$$

where $K_{s}$ is a non-zero constant. It then follows from (21) that $h^{2}$ is of the form

$$
h^{2} \rightarrow \frac{1}{n^{2}}\left[\left(K_{s} r^{s} P_{s}(\cos \theta)\right)^{2}+\left(K r^{m} P_{m}^{n}(\cos \theta)\right)^{2}\right], s \leq m .
$$

as $r \rightarrow 0$. Equation (24) shows that $h^{2}$ is a regular function at $z_{\mathrm{mo}}$ and that

$$
h^{2}(r, 0)=h^{2}(r, \pi), \quad r \neq 0 .
$$

Thus the field equations require that $h^{2}$ be symmetric about any point on the $z$-axis where it vanishes. It is indeed possible for a single point charge distribution to satisfy this. However, now consider a charge which is not distributed at a single point on the z-axis. According to the previous discussion of the topology, $h^{2}$. in this case, must vanish along a 
finite section of the z-axis. On the z-axis in a neighbourhood of an end point $z_{0}$ of such a section, $h^{2}=0$ inside $z_{m}$. and $h^{2} \neq 0, b^{2}=n^{2} \neq 0$ outside. Thus $h^{2}$ can not be symmetric about $z_{m}$. Therefore equation (25) rules out all but the single point charge distribution.

Let us now assume that the charge distribution is concentrated at a single point (superimposed monopoles), which we take to be the origin. The equations derived above for $z_{0}$ are still valid, so that near the origin we have tholeading behaviour

$$
\begin{aligned}
& h k \rightarrow K r^{m} P_{m}^{n}(\cos \theta), m \geq n, \\
& h b \rightarrow K K_{s} r^{s} P_{s}(\cos \theta), m \geq s \geq 1 . \\
& h \rightarrow \frac{1}{n} r^{s}\left|K_{s} P_{s}(\cos \theta)\right|, \text { for } s<m . \\
& h \rightarrow \frac{1}{n} r^{m}\left\{\left(K K_{m} P_{m}(\cos \theta)\right)^{2}+\left(K P_{m}^{n}(\cos \theta)\right)^{2}\right\}^{1 / 2} \\
& \quad \text { for } s=m .
\end{aligned}
$$

where the condition $s \leq m$ comes from the fact that if $s>m$, we have $b \rightarrow 0$ and hence no topological charge. The most natural case (and possibly only stable case) is $m=n$, and then we have

$$
\begin{aligned}
& h k \rightarrow K \rho^{n} . \\
& h b \rightarrow K{ }_{s} r^{s} P_{s}(\cos \theta) . \\
& h \rightarrow \frac{1}{n} r^{s}\left|K_{s} P_{s}(\cos \theta)\right|, \text { for } s<m . \\
& h \rightarrow \frac{1}{n} r^{n}\left\{\left(K_{n} P_{n}(\cos \theta)\right)^{2}+K^{2} \rho^{2 n}\right\}^{1 / 2} . . \\
& \text { for } s=m .
\end{aligned}
$$

It has been pointed out by $P$. Rossi that when $s=1$ the behaviour at the origin can be determined more precisely, namely as

$$
h^{2}=\left(\sum_{1=1}^{n} k_{1} \frac{r^{1}}{n} P_{1}(\cos \theta)\right)^{2} .
$$




$$
b^{2}=\left(n+\sum_{1=1}^{n} \frac{\sin ^{2} \theta r^{1+1}}{n(1+1)} k_{1} P_{1}^{\prime}(\cos \theta)\right)^{2} .
$$

This result can be understood in our context, and be slightly generalized, by noting that if $s<m$ then $k \sim r^{m-s}$ and hence from the field equations (3) - (5)

$$
b_{, \alpha}+\rho \in_{\alpha \beta} h_{, \beta}=o\left(r^{2 m-2 s-1}\right) \text {. }
$$

which implies that, to the order shown, there exists a harmonic function

$$
U=\sum_{1=1}^{n+1} \gamma_{1} r^{1} P_{1}(\cos \theta)
$$

such that

$$
h^{2}=\left(\frac{\partial u}{\partial z}\right)^{2} \text {, and } b^{2}=\rho^{2}\left(\frac{\partial u}{\partial \rho}\right)^{2} \text {. }
$$

Alternatively, we may note that to the order in which $k$ (and therefore $r u_{\alpha}$ ) may be neglected the field equations (7) reduce to the two equations $\Delta h=0$ and (16) and that these two equations are identically satisfied by (31) if $U$ is harmonic.

A further symmetry which is carried by the field equations (3) - (5) is invariance under reflexion in the (xy)-plane, where $h, k$ are even and $b$ is odd with respect to this reflexion (in agreement with the boundary conditions as $r \rightarrow \infty$ ). If we impose this condition on the fields then all the integers, $m, s, n$ above must be odd. In particular, only odd topological charges $n$ are permitted. Since this symmetry agrees naturally with the equation and boundary conditions, it suggests that even topological charges might not be allowed [7] (under the assumptions of axial symmetry).

Finally we should mention that the results mentioned here are only very slightly modified when the assumption of mirror 
symmetry is removed. Indeed, apart from the complication due to the introduction of four new fields, the results go through for axial symmetry alone. This generalization and a more detalled treatment of the above results, will be given in a forthcoming paper.

\section{References}

[1] G. 't Hooft, Nucl. Phys, B79, 276 (1976)

A. Polyakov, JETP letters, 20, 194 (1974)

[2] E. Bogomolny, Sov. J. Nucl. Phys. 24, 861 (1976)

P. Houston, L. O'Raifeartaigh. Proc. Seminar on Group Th. and Quant. Fields, Zvenigorod, Moscow, Nov. 1979. DIAS-STP-79-35

N. Manton, Nucl. Phys. B135, 319 (1978)

[4] S. Adler, Private Communication

5] Arafune, P. Freund, C. Goebel, J. Math. Phys, 16. 433 (1975)

[6] P. Houston, L. O'Raifeartaigh, DIAS-STP-80-14 (1980) See also P.S. Jang, S.Y. Park, K.C. Wali, Phys. Rev. D17. 1641 (1978)

[7] See also J. Madore, Gen. Rel, and Grav. 8, 655 (1977) 